\title{
Visual and autorefraction outcomes following toric intraocular lens insertion without calculation of posterior corneal astigmatism in the UK National Health Service
}

\author{
Alasdair Warwick $\mathbb{D}^{1} \cdot$ Alastair Porteous $^{1} \cdot$ Valerie P. J. Saw $^{1,2}$ \\ Received: 1 July 2019 / Revised: 17 January 2020 / Accepted: 17 January 2020 / Published online: 29 January 2020 \\ (c) The Author(s), under exclusive licence to The Royal College of Ophthalmologists 2020
}

\begin{abstract}
Objective To ascertain visual and refractive outcomes following toric intraocular lens (IOL) implantation in the UK National Health Service (NHS) without posterior corneal astigmatism calculation, with multiple surgeons of different grades, pooled input and output pathways and autorefraction as the refractive outcome measure.

Methods Preoperative and 1-month post-operative data were analysed retrospectively in 114 eyes (95 patients) receiving a toric IOL between 2014 and 2016 at Imperial College NHS Trust. Preoperative keratometric astigmatism was $\geq 2$ dioptres (D).

Results Mean preoperative best-corrected visual acuity (BCVA) was $0.50 \log$ MAR $( \pm 0.46)$, improving to a mean uncorrected VA (UCVA) of $0.35 \log$ MAR $( \pm 0.36)$ postoperatively $(p<0.001)$ with $65 \%$ of eyes attaining a UCVA $\leq 0.30$ $\log$ MAR. Excluding 33 eyes with pre-existing visual comorbidities and one targeting monovision, mean post-operative UCVA was $0.24 \log$ MAR $( \pm 0.29)(p<0.001)$, and $85 \%$ had UCVA $\leq 0.30 \log$ AAR, $62 \%$ UCVA $\leq 0.20 \operatorname{logMAR}$. Mean refractive astigmatism improved from $3.04 \mathrm{D}( \pm 1.46)$ to $1.36 \mathrm{D}( \pm 1.13)(p<0.001)$. In total, $52 \%$ of eyes had post-operative refractive astigmatism $\leq 1.00 \mathrm{D}$. The Alpins correction index was $1.05( \pm 0.22)$, indicating a tendency to overcorrect. Toric IOL misalignment was noted in two eyes, and two cases of posterior capsule rupture were converted to a non-toric IOL. Conclusions Visual outcomes of toric IOL implantation in our pooled pathway are comparable to single-surgeon case series where posterior corneal astigmatism has not been accounted for. However, with 1-month post-operative autorefraction, only $52 \%$ of eyes had $\leq 1 \mathrm{D}$ refractive astigmatism, which is lower than previously published series, but may be standard for 1 -month autorefraction outcomes.
\end{abstract}

\section{Introduction}

Cataract surgery is the most common surgery performed by ophthalmologists and can significantly improve the patient's quality of life [1]. There is increasing emphasis on optimising

This paper was presented at European Society of Cataract and Refractive Surgeons (ESCRS) Conference 22-26 September 2018, Vienna, Austria and British Society for Refractive Surgery (BSRS) Conference 23-24 June 2018, Wishaw, UK.

Valerie P. J. Saw

v.saw@nhs.net

1 Imperial College Healthcare NHS Trust, Western Eye Hospital, 153-173 Marylebone Road, London NW1 5QH, United Kingdom

2 Institute of Ophthalmology, University College London, 11-43 Bath St, London EC1V 9EL, United Kingdom refractive outcomes to decrease post-operative dependency on spectacles. Approximately $20 \%$ of patients have 1.50 dioptres (D) or greater astigmatism preoperatively, and residual post-operative astigmatism is a significant determinant of reduced post-operative uncorrected visual acuity (UCVA) $[2,3]$. Inserting a toric intraocular lens (IOL) during cataract surgery offers a safe, single-step method for correcting regular astigmatism, and is more effective and reliable in reducing post-operative spectacle dependency than relaxing incisions $[4,5]$.

Whilst toric IOLs are now widely used in routine cataract surgery, there are few large series-published outcomes of surgery carried out in a UK National Health Service (NHS) setting with multiple surgeons of different levels of surgical experience (consultant, fellow or trainee), and different levels of surgically induced astigmatism, where the surgeon who orders the toric implant may not be the surgeon who implants the lens due to pooled input and output pathways, 
and where post-operative autorefraction outcomes at 1 month (rather than manifest refraction by an optometrist) are standard. The aim of this study was therefore to report visual and refractive outcomes following toric IOL insertion during cataract surgery in this setting. Comparison with previously published results is discussed.

\section{Subjects and methods}

This was a retrospective study of all eyes undergoing surgery for age-related cataract with toric IOL insertion at the Imperial College NHS Trust at the Western Eye Hospital, UK, between 1st November 2014 and 1st November 2016. Patients were identified from the toric IOL order logbook, and data were collected from both paper and electronic hospital records. Complications, including cases where an intraoperative complication prohibited implantation of the planned toric IOL, are reported. All eyes had preoperative keratometric regular astigmatism of $\geq 2 \mathrm{D}$, as per the local protocol. All study participants had given consent for their data to be analysed for audit purposes, and approval for this audit was given by the Imperial College NHS Healthcare Trust Clinical Governance Committee (audit registration number 858974).

\section{Preoperative assessment}

Preoperative assessment included Snellen best-corrected distance visual acuity (BCVA), UCVA, refraction, biometry and a dilated fundus examination. Autorefraction was performed with a Canon RK-5 autorefractor (Canon). Keratometry and axial length were obtained by using an IOLMaster (Zeiss). Ultrasound axial-length measurements were recorded for eyes where it was not possible to obtain reliable optical readings. If $\geq 2 \mathrm{D}$ keratometric astigmatism was detected at preoperative assessment, then corneal topography was performed, and a senior ophthalmologist or corneal specialist was consulted to assess suitability for toric IOL insertion. Topography was performed using either a Pentacam (Oculus) or Orbscan (Bausch and Lomb) to confirm regular corneal astigmatism.

\section{Intraocular lens}

A T-flex 623T 1-piece hydrophilic acrylic closed-loop haptic toric IOL (Rayner Intraocular Lenses Ltd., East Sussex, England) was used for all procedures. The required IOL power was calculated by inputting biometry values into the IOL manufacturer's calculator, available online (https://www.raytrace.rayner.com/). This toric calculator estimates the effective lens position, but does not take into account posterior corneal astigmatism.

\section{Surgical technique}

Corneal marking to guide toric IOL orientation was performed at the slit lamp immediately prior to surgery, according to individual surgeon preference. A standard phacoemulsification and IOL insertion technique was performed using a 2.75-mm clear corneal incision. Surgery was performed by consultant ophthalmologists, fellows or ophthalmology trainee doctors under consultant supervision. Standard regimens of antibiotic and steroid drops were administered postoperatively as per the local protocol.

\section{Post-operative assessment}

Patients received a follow-up appointment at 1 month to assess visual acuity and autorefraction. A dilated slit-lamp examination was also performed to check for post-operative complications including toric IOL misalignment.

\section{Vector analysis}

The Alpins method of vector analysis was used to evaluate the effectiveness of astigmatism correction using toric IOLs [6]. Post-operative refractive astigmatism was converted to the corneal plane, using a vertex distance of $12 \mathrm{~mm}$, and compared with preoperative keratometric astigmatism.

\section{Statistics}

Snellen visual acuity measurements were converted to $\log$ MAR equivalents for the purpose of analysis. Statistical analysis was performed using GraphPad Prism7 (GraphPad Software, La Jolla, California, USA), and $p<0.05$ was considered statistically significant. Parametric variables were compared using the student $t$ test, and the chi-square test was used for categorical variables. Continuous data are presented as mean ( \pm standard deviation) unless stated otherwise. Numbers in square brackets denote the number of eyes with available data for the parameter of interest.

\section{Results}

One-hundred and twenty-one cataract operations (1.3\% of all cataract operations), involving toric intraocular lens insertion, were identified for the studied period. In total, 114 eyes (94\%) of 95 patients had available notes, of which 33 eyes (29\%) had visually significant comorbidities. One eye aimed for monovision $(-1.40 \mathrm{D}$ spherical equivalent $(\mathrm{SE}))$, and all other cases aimed for a refractive outcome between emmetropia and $-0.50 \mathrm{D}$. In total, $66(58 \%)$ operations were performed by consultant ophthalmologists, 22 (19\%) by senior ophthalmology fellows and $26(23 \%)$ by ophthalmology 
Table 1 Baseline preoperative characteristics for the entire cohort. Values are presented as mean \pm standard deviation (range) or percentages.

\begin{tabular}{ll}
\hline Parameter & $\begin{array}{l}\text { Mean } \pm \text { standard } \\
\text { deviation }\end{array}$ \\
\hline Age [114] & 70 years \pm 13.1 \\
Male [114] & $45 \%$ \\
Operated right eye [114] & $55 \%$ \\
UCVA [42] & $0.82 \operatorname{logMAR} \pm 0.56$ \\
BCVA [111] & $0.50 \operatorname{logMAR} \pm 0.46$ \\
Axial length [112] & $23.55 \mathrm{~mm} \pm 1.17$ \\
High myopia ${ }^{\text {a }}[112]$ & $5 \%$ \\
Refractive astigmatism [94] & $3.04 \mathrm{D} \pm 1.46$ \\
Keratometric astigmtism [114] & $3.33 \mathrm{D} \pm 1.16$ \\
Spherical equivalent of preoperative & $-0.13 \mathrm{D} \pm 3.84$ \\
refraction [94] &
\end{tabular}

UCVA uncorrected visual acuity, BCVA best-corrected visual acuity, $D$ dioptres.

${ }^{a}$ High myopia was defined as axial length $\geq 26 \mathrm{~mm}$. Numbers in square brackets denote the number of eyes for which data were available.

trainee registrars. The mean cylindrical power of implanted toric IOLs was $4.13 \mathrm{D}( \pm 1.08)$ and ranged from 2 to $6 \mathrm{D}$. Baseline preoperative characteristics for the entire cohort are displayed in Table 1.

\section{Visual acuity outcomes}

The mean post-operative BCVA and UCVA values were $0.20 \log$ MAR $( \pm 0.34)[n=106]$ and $0.35 \log$ MAR $( \pm 0.36)$ $[n=92]$, respectively. Both improved significantly compared with preoperative VA $(p<0.001)$. The proportion of eyes that achieved post-operative UCVA $\leq 0.30 \log$ MAR (>6/12 Snellen) was $65 \%$.

When excluding 33 eyes with pre-existing visually significant comorbidities (including advanced glaucoma, moderate-severe diabetic retinopathy, macula pathology and previous ocular surgery) and one eye aiming for monovision ( $-1.40 \mathrm{D} \mathrm{SE})$, the mean post-operative BCVA and UCVA values were $0.12 \operatorname{logMAR}( \pm 0.27)[n=76$ ] and 0.24 $\log$ MAR $( \pm 0.29)[n=66]$, respectively. Both improved significantly compared with preoperative VA $(p$ $<0.001)$. The proportion of these eyes who achieved postoperative UCVA $\leq 0.30 \log$ MAR ( $>6 / 12$ Snellen) was $85 \%$. The proportion of eyes who achieved post-operative UCVA $\leq 0.20 \log$ MAR ( $>6 / 9$ Snellen) was $62 \%$.

\section{Residual refractive astigmatism}

The mean post-operative SE was $-0.53 \mathrm{D}( \pm 0.94)[n=85]$. The mean post-operative refractive astigmatism was $1.36 \mathrm{D}$ $( \pm 1.13)[n=85]$, and was significantly improved compared with preoperative refractive astigmatism $(p<0.001)$.
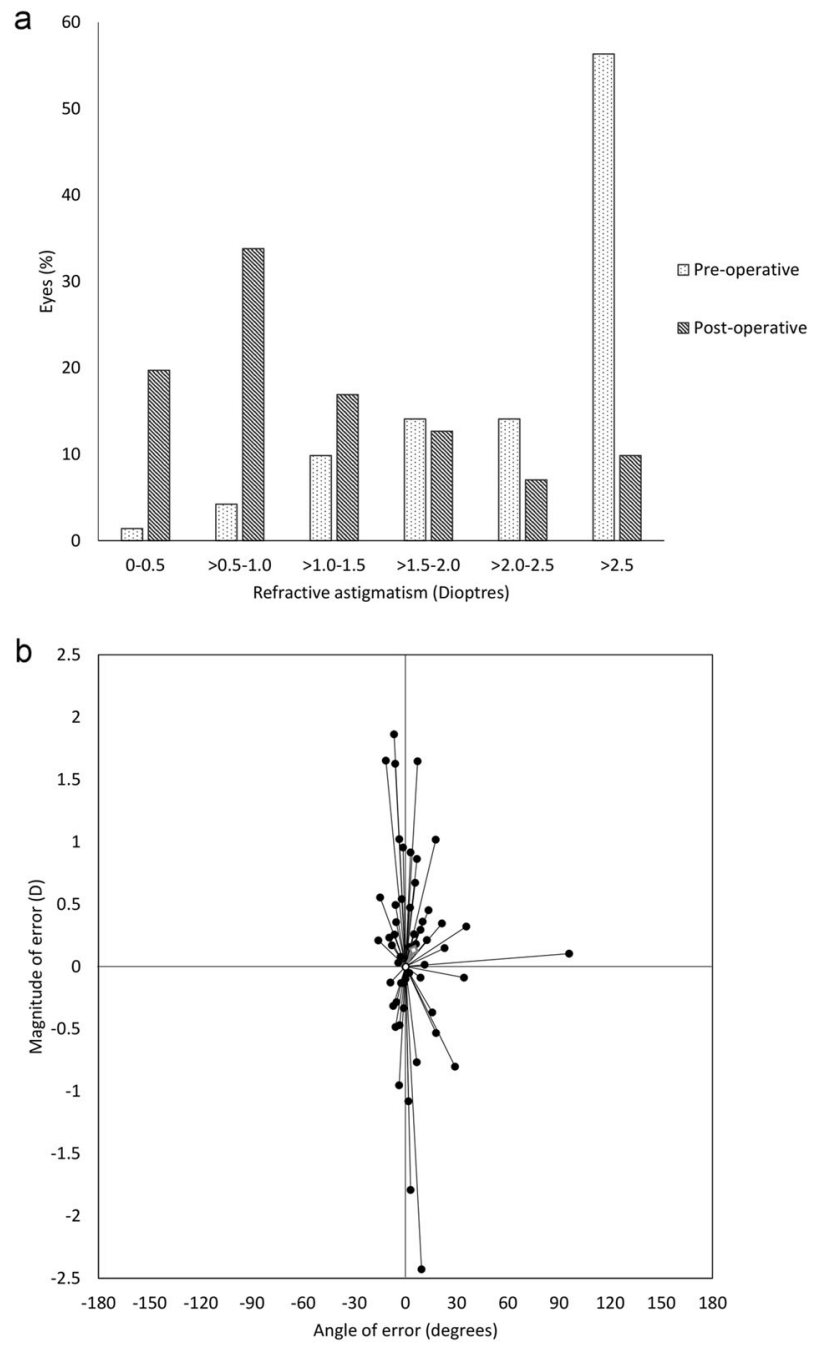

Fig. 1 a Proportion of eyes with different degrees of refractive astigmatism pre- and postoperatively. Note that refractive astigmatism preoperatively in some cases was $<2.00 \mathrm{D}$ but a toric IOL was implanted because keratometric astigmatism was $\geq 2.00 \mathrm{D}$. b Vector analysis showing the magnitudes and angles of error for individual eyes (black dots). The average result is depicted by the red diamond.

Figure 1a compares the proportion of eyes with different levels of refractive astigmatism pre- and postoperatively. No significant associations were found between residual post-operative refraction and age, gender and surgeon grade. There was also no evidence of any significant correlation between preoperative keratometric astigmatism and post-operative refractive astigmatism (Table 2).

For 70 eyes with complete pre- and post-operative refractive data the mean reduction in astigmatism was $1.69 \mathrm{D}( \pm 1.60)$. Astigmatism on autorefraction increased at 1 month postoperatively in nine eyes. For one eye, the IOL was found to be misaligned, and one other had required a corneal suture. No clear reason for the increased astigmatism was documented for the remaining seven eyes, however all had post-operative UCVA of $0.30 \log$ MAR or better. 
Table 2 Evaluation of preoperative keratometric astigmatism and post-operative refractive astigmatism.

\begin{tabular}{lll}
\hline $\begin{array}{l}\text { \% of eyes } \\
(n / \text { total })\end{array}$ & Pre-op keratometric astigmatism & Post-op refractive astigmatism mean (SD) \\
\hline $54 \%(46 / 85)$ & $2.00-2.99 \mathrm{D}$ & $1.21( \pm 1.18)$ \\
$26 \%(22 / 85)$ & $3.00-3.99 \mathrm{D}$ & $1.46( \pm 1.04)$ \\
$20 \%(17 / 85)$ & $\geq 4.00 \mathrm{D}$ & $1.65( \pm 1.09)$ \\
\hline$\%$ of eyes & Post-operative refractive astigmatism & Preoperative keratometric astigmatism \\
$(n /$ total $)$ & & mean $(\mathrm{SD})$ \\
\hline $34 \%(29 / 85)$ & $<1.00 \mathrm{D}$ & $2.92( \pm 0.73)$ \\
$45 \%(38 / 85)$ & $1.00-2.00 \mathrm{D}$ & $3.30( \pm 0.95)$ \\
$45 \%(38 / 85)$ & $2.00-3.00 \mathrm{D}$ & $3.31( \pm 1.00)$ \\
\hline
\end{tabular}

Values are presented as mean \pm standard deviation or percentages.

$D$ dioptres.

No correlation between the two parameters was evident.

\section{Refractive predictability}

Seventy-one eyes had complete data for predicted postoperative refraction and actual post-operative refraction. In total, $27 \%$ were within $\pm 0.50 \mathrm{D}$ of the predicted postoperative astigmatism, and $59 \%$ were within $\pm 1 \mathrm{D}$ of the predicted post-operative astigmatism. Mean deviation from the predicted post-operative astigmatism at 1 month was $1.16 \mathrm{D}( \pm 1.01)$.

In total, $35 \%$ were within $\pm 0.50 \mathrm{D}$ of the predicted postoperative SE, and $76 \%$ were within $\pm 1 \mathrm{D}$ of the predicted post-operative SE. Mean deviation from the predicted postoperative SE was $-0.27 \mathrm{D}( \pm 0.96)$.

\section{Vector analysis}

Vector analysis was performed for 55 eyes with complete preoperative keratometry, predicted post-operative astigmatism and actual post-operative refractive astigmatism data (Table 3). The magnitude of error of +0.13 and correction index of 1.05 indicate a general tendency to overcorrect astigmatism. The angle of error of $+5^{\circ}$ indicates that the achieved correction tended to be counterclockwise to its intended axis. This is displayed graphically in Fig. $1 b$.

\section{Complications}

Toric IOL misalignment was noted in two eyes postoperatively, one of whom underwent IOL repositioning with a subsequent improvement in UCVA from 0.78 logMAR to $0.30 \log$ MAR. The other patient declined further surgery. There were two cases of posterior capsular rupture necessitating conversion to non-toric monofocal IOL insertion. Other complications included post-operative cystoid macula oedema in three eyes (two of which had diabetes), two cases of post-operative uveitis and one
Table 3 Vector analysis by Alpins method [6] showing the effectiveness of astigmatism correction by toric intraocular lenses.

\begin{tabular}{lll}
\hline Characteristic & This study & Alio 2010 \\
\hline $\begin{array}{l}\text { Target-induced astigmatism, } \\
\text { mean (SD) }\end{array}$ & $3.24 \mathrm{D}(1.00)$ & $4.54( \pm 2.72)$ \\
$\begin{array}{l}\text { Surgically induced astigmatism, } \\
\text { mean (SD) }\end{array}$ & $3.37 \mathrm{D}(1.17)$ & $4.18( \pm 2.66)$ \\
$\begin{array}{l}\text { Difference vector, mean (SD) } \\
\text { Magnitude of error, mean (SD) }\end{array}$ & $1.23 \mathrm{D}(0.94)$ & $0.47( \pm 0.61)$ \\
Angle of error, mean (SD) & $5^{\circ}(17)$ & $-1.2^{\circ}( \pm 1.9)$ \\
Absolute angle of error, mean $(\mathrm{SD})$ & $10^{\circ}(14)$ & - \\
Correction index & 1.05 & 0.91 \\
Index of success & 0.38 & 0.11 \\
\hline
\end{tabular}

$S D$ standard deviation, $D$ dioptres.

Results from Alio et al. are also shown for comparison [8].

intraoperative vitreous haemorrhage that developed after the toric IOL had been successfully inserted.

\section{Discussion}

The first toric IOL was designed in 1993 and since then numerous studies have shown toric IOL insertion to be a safe and effective method of astigmatic correction [7]. A recent meta-analysis by Kessel et al. found toric IOLs to provide better UCVA, greater spectacle independence and less residual astigmatism than non-toric IOLs combined with relaxing incisions [5]. The authors did however comment that their conclusions were limited to a range of 0.75-3.00 D preoperative astigmatism. Indeed, the majority of toric IOL studies have only included relatively low levels of preoperative astigmatism, and there are comparatively few reported outcomes for moderate-to-high preoperative astigmatism ( $>3 \mathrm{D})$ [5]. 


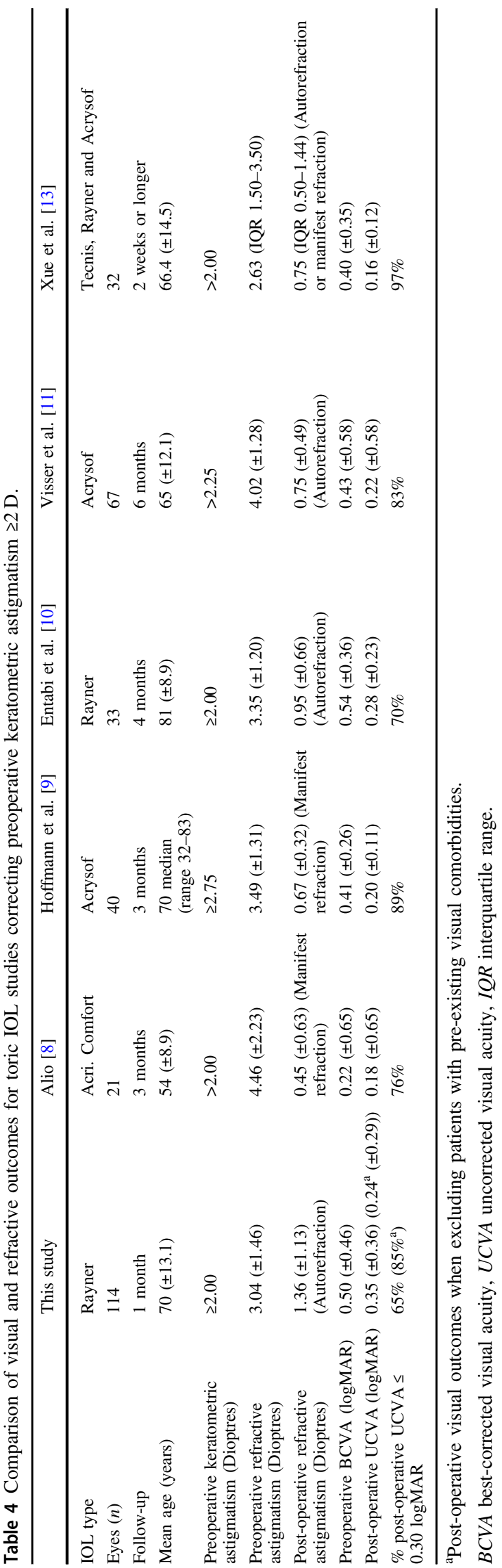

Previous studies have also excluded eyes with visually significant comorbidities, which is not reflective of realworld clinical practice. In the UK National Health Service, there are multiple surgeons with different levels of experience (Consultant, Senior Fellow and Trainee) implanting toric IOLs. Whilst the surgical equipment used is similar for all cases including keratome blade size, and a uniform protocol for toric IOL ordering is in place, different surgeons have different levels of surgically induced astigmatism and due to pooled input and output pathways, the surgeon ordering the toric IOL may not be the surgeon who implants the toric IOL. There is significant variability in this process, and this study evaluates how outcomes in this setting compare with published single-/dual-surgeon series.

Evaluating toric IOL outcomes in this setting are particularly relevant, given that the Trust may not receive a higher tariff for cataract surgery with toric IOL compared with a non-toric IOL, despite the additional preoperative corneal topography test, higher cost of the toric IOL, increased administrative time spent on toric IOL calculation and ordering, increased operating time and use of additional marking instruments.

While thresholds may vary across different UK units, toric IOLs are commonly considered for eyes that have $\geq 2 \mathrm{D}$ of regular keratometric astigmatism at pre-assessment. In Table 4 our results are compared with those from previous toric IOL studies which also included eyes with preoperative keratometric astigmatism of $\geq 2.00 \mathrm{D}$ [8-11]. The mean post-operative UCVA for our entire cohort was $0.35 \log$ MAR, which was significantly improved from preoperative BCVA, and $65 \%$ achieved a post-operative UCVA of $\leq 0.30 \log$ MAR ( $>6 / 12$ Snellen). This included a number of eyes with pre-existing visual comorbidities, whereas the other listed studies excluded such eyes. When excluding these eyes from our cohort the mean UCVA was $0.24 \log$ MAR and $85 \%$ achieved a post-operative UCVA of $\leq 0.30 \log \mathrm{MAR}$, which is more similar to other reports (Table 3). These findings are also comparable to results from the Royal College of Ophthalmologists' National Ophthalmology Database study of cataract surgery, which looked at all cataract operations performed in the UK between 2006 and 2010, reporting that the mean postoperative UCVA for eyes without ocular co-pathology and the proportion of these eyes achieving $\leq 0.30 \log$ MAR postoperative UCVA were $0.27 \operatorname{logMAR}$ and $81 \%$ respectively [12]. A recent small series of 32 eyes using three types of toric IOLs in the UK National Health Service found a mean post-operative logMAR UCVA 0.00-0.48 [13].

Despite comparable visual outcomes to those in the literature, the proportion of our patients achieving a postoperative SE within $\pm 0.50 \mathrm{D}$ and $\pm 1 \mathrm{D}$ of predicted postoperative SE was relatively low (35\% and $76 \%$, respectively). This is below previously suggested benchmarks $(55 \%$ within 
$0.50 \mathrm{D}$ and $85 \%$ within $1.00 \mathrm{D}$ respectively) for postoperative SE following NHS cataract surgery [14], although audits of post-operative outcomes for non-toric IOLs in our Trust (unpublished) do meet these standards. Furthermore, the mean residual post-operative astigmatism for our cohort was relatively high at $1.36 \mathrm{D}$. While the majority of eyes $(59 \%)$ were within $\pm 1.00 \mathrm{D}$ of the predicted post-operative astigmatism, only $27 \%$ were within $\pm 0.50 \mathrm{D}$.

The positive magnitude of error and correction index $>1$ indicate a general tendency to have overcorrected astigmatism. There were no significant associations between preoperative factors such as preoperative astigmatism and postoperative refractive outcome. The disparity between postoperative UCVA and refractive outcomes may be in part due to inaccurate autorefraction readings. In support of this, of the nine eyes for which astigmatism was found to be higher postoperatively, no apparent cause was found in seven, and all these achieved UCVA $\leq 0.30 \log$ MAR. Interestingly however, in unpublished real-world data from another large UK unit, also using autorefraction outcomes, the mean residual post-operative astigmatism was found to be similar to our own (1.15 D) [15].

An important cause of refractive surprise following toric IOL implantation is lens misalignment. Misalignment by $10^{\circ}$ results in a $35 \%$ residual error in the magnitude of astigmatism and misalignment by $30^{\circ}$ eliminates any corrective effect $[16,17]$. In our study the angle of error was positive, meaning that the achieved correction tended to be counterclockwise to its intended axis, while the absolute angle of error was $10^{\circ}$ on average. Toric misalignment was noted in two eyes $(1.8 \%)$ at their post-operative follow-up. Findings from the recent metaanalysis by Kessel et al. showed that 6 of $554(1.1 \%)$ eyes required a second surgical procedure for toric IOL realignment [5].

A further factor to consider in improving toric IOL outcomes is use of newer toric calculators (e.g. the Barrett toric calculator) that take into account both posterior and anterior corneal curvature, which appear to be more accurate [18]. The Acrysof online toric calculator has recently been modified to include the Barrett toric calculation. The Rayner online toric calculator currently uses the estimated effective lens position to calculate the IOL cylinder power at the corneal plane, and does not estimate net corneal astigmatism, which is influenced by both posterior and anterior corneal curvature.

In summary, we report real-world outcomes for toric IOL implantation by multiple surgeons in pooled NHS pathways, showing that visual outcomes are similar to single-/dual-surgeon case series. Whilst refractive outcomes using 1-month autorefraction data show lower levels of correction compared with previous publications using subjective refraction at 3 months, these data may be used as a reference against which other units could compare their own outcomes. Further real-world studies of toric IOL insertion for moderate-high preoperative astigmatism would help to better evaluate efficacy and safety.

\section{Summary}

\section{What was known before}

- Toric intraocular lens implantation in a single-/dualsurgeon setting with a single input and output pathway results in safe and effective correction of astigmatism, as assessed by post-operative subjective refraction.

\section{What this study adds}

- Real-world NHS toric intraocular lens implantation in a multiple-surgeon teaching hospital setting with pooled input and output pathways results in visual outcomes comparable to single-surgeon case series.

- Real-world NHS refractive outcomes using 1-month post-operative autorefraction show higher levels of postoperative astigmatism.

\section{Compliance with ethical standards}

Conflict of interest The authors declare that they have no conflict of interest.

Publisher's note Springer Nature remains neutral with regard to jurisdictional claims in published maps and institutional affiliations.

\section{References}

1. Lamoureux EL, Fenwick E, Pesudovs K, Tan D. The impact of cataract surgery on quality of life. Curr Opin Ophthalmol. 2011;22:19-27.

2. Ostri C, Falck L, Boberg-Ans G, Kessel L. The need for toric intra-ocular lens implantation in public ophthalmology departments. Acta Ophthalmol. 2015;93:e396-7.

3. Behndig A, Montan P, Stenevi U, Kugelberg M, Zetterstrom C, Lundstrom M. Aiming for emmetropia after cataract surgery: Swedish National Cataract Register study. J Cataract Refract Surg. 2012;38:1181-6.

4. Visser N, Beckers HJ, Bauer NJ, Gast ST, Zijlmans BL, Berenschot TT, et al. Toric vs aspherical control intraocular lenses in patients with cataract and corneal astigmatism: a randomized clinical trial. JAMA Ophthalmol. 2014;132:1462-8.

5. Kessel L, Andresen J, Tendal B, Erngaard D, Flesner P, Hjortdal $\mathrm{J}$. Toric intraocular lenses in the correction of astigmatism during cataract surgery: a systematic review and meta-analysis. Ophthalmology. 2016;123:275-86. 
6. Alpins NA. A new method of analyzing vectors for changes in astigmatism. J Cataract Refract Surg. 1993;19:524-33.

7. Kaur M, Shaikh F, Falera R, Titiyal JS. Optimizing outcomes with toric intraocular lenses. Indian J Ophthalmol. 2017;65:1301-13.

8. Alio JL, Agdeppa MC, Pongo VC, El Kady B. Microincision cataract surgery with toric intraocular lens implantation for correcting moderate and high astigmatism: pilot study. J Cataract Refract Surg. 2010;36:44-52.

9. Hoffmann PC, Auel S, Hutz WW. Results of higher power toric intraocular lens implantation. J Cataract Refract Surg. 2011; 37:1411-8.

10. Entabi M, Harman F, Lee N, Bloom PA. Injectable 1-piece hydrophilic acrylic toric intraocular lens for cataract surgery: efficacy and stability. J Cataract Refract Surg. 2011;37:235-40.

11. Visser N, Ruiz-Mesa R, Pastor F, Bauer NJ, Nuijts RM, MontesMico R. Cataract surgery with toric intraocular lens implantation in patients with high corneal astigmatism. J Cataract Refract Surg. 2011;37:1403-10.

12. Day AC, Donachie PH, Sparrow JM, Johnston RL, Royal College of Ophthalmologists' National Ophthalmology Database. The Royal College of Ophthalmologists' National Ophthalmology Database study of cataract surgery: report 1 , visual outcomes and complications. Eye. 2015;29:552-60.
13. Xue K, Jolly JK, Mall SP, Haldar S, Rosen PH, MacLaren RE. Real-world refractive outcomes of toric intraocular lens implantation in a United Kingdom National Health Service setting. BMC Ophthalmol. 2018;18:30.

14. Gale RP, Saldana M, Johnston RL, Zuberbuhler B, McKibbin M. Benchmark standards for refractive outcomes after NHS cataract surgery. Eye. 2009;23:149-52.

15. Khan S, Little B, Maurino V. Outcomes of 272 eyes with Acrysof IQ toric intraocular lens following routine cataract surgery in the National Health Service, United Kingdom. http://www.escrs.org/Copenhagen2016/programme/free-papersdetails.asp $\mathrm{id}=26981 \&$ day $=0$. Accessed 2 April 2019.

16. Felipe A, Artigas JM, Diez-Ajenjo A, Garcia-Domene C, Alcocer P. Residual astigmatism produced by toric intraocular lens rotation. J Cataract Refract Surg. 2011;37:1895-901.

17. Visser N, Bauer NJ, Nuijts RM. Toric intraocular lenses: historical overview, patient selection, IOL calculation, surgical techniques, clinical outcomes, and complications. J Cataract Refract Surg. 2013;39:624-37.

18. Abulafia A, Barrett GD, Kleinmann G, Ofir S, Levy A, Marcovich AL, et al. Prediction of refractive outcomes with toric intraocular lens implantation. J Cataract Refract Surg. 2015;41:936-44. 